\title{
Suppression mechanism of the ferroelectric polarization in multiferroic tetragonal perovskite $\mathrm{Sr}_{1 / 2} \mathrm{Ba}_{1 / 2} \mathrm{MnO}_{3}$
}

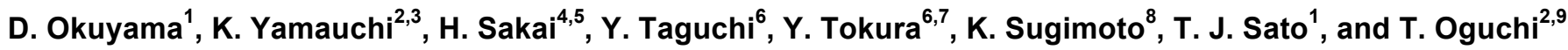 \\ ${ }^{I} I M R A M$, Tohoku Univ., Sendai, Japan, ${ }^{2}$ ISIR-SANKEN, Osaka University, Ibaraki, Japan, ${ }^{3}$ ESICB, Kyoto University, Kyoto, Japan, \\ ${ }^{4}$ Department of Physics, Osaka University, Toyonaka, Japan, ${ }^{5}$ PRESTO, Japan Science and Technology Agency, Kawaguchi, Japan, \\ ${ }^{6}$ RIKEN Center for Emergent Matter Science, Wako, Japan, ${ }^{7}$ Tokyo College and Department of Applied Physics, University of Tokyo, \\ Tokyo, Japan, ${ }^{8}$ JASRI SPring-8, Hyogo, Japan, ${ }^{9}$ CSRN, Graduate School of Engineering Science, Osaka University, Toyonaka, Japan
}

\section{okudaisu@tohoku.ac.jp}

Ferroelectricity for the ferroelectric perovskite oxides $(\mathrm{ABO})_{3}$ have been investigated for decades [1]. In the ferroelectric BaTiO $\mathrm{B}_{3}$, it is well known that the electronic $p$ - $d$ hybridization between empty $d$ orbitals of titanium and filled $2 p$ orbitals of oxygen causes a large ferroelectric polarization [2]. In contrast, magnetic-ordering-induced ferroelectric materials (multiferroics) have also been extensively investigated since a large nonlinear magnetoelectric effect was found in the perovskite $\mathrm{TbMnO}_{3}[3]$. In tetragonal perovskite $\mathrm{BaMnO}_{3}$, it is proposed that a large ferroelectricity is induced by the distortion of the $\mathrm{Mn}$ and $\mathrm{O}$ ions originating from the $p$ - $d$ hybridization in the paramagnetic phase. Since the magnetic $\mathrm{Mn}^{4+}$ ion contributes to the emergence of the ferroelectricity, a large magnetoelectric effect is expected. Sakai et al. grew the tetragonal perovskite $\mathrm{Sr}_{1 / 2} \mathrm{Ba}_{1 / 2} \mathrm{MnO}_{3}$ [4]. A large reduction in the ferroelectric polarization is observed below the magnetic transition temperature. The reduction mechanism of the ferroelectric polarization should be unveiled by dividing the ferroelectric polarization into the respective contributions from the $p$ - $d$ hybridization and that from the magnetic interaction.

Here, we report the atomic displacements in the ferroelectric and multiferroic phases of the tetragonal perovskite $\mathrm{Sr}_{1 / 2} \mathrm{Ba}_{1 / 2} \mathrm{MnO}_{3}$ determined by the crystal-structure analyses [5]. Using a first-principles calculation based on accurate crystal-structure parameters, we quantitatively elucidate the suppression mechanism of the ferroelectric polarization in the multiferroic phase. The synchrotron $\mathrm{x}$-ray diffraction experiments were carried out in the ferroelectric $(T=225 \mathrm{~K})$ and multiferroic $(T=50 \mathrm{~K})$ phases of the tetragonal perovskite $\mathrm{Sr}_{1 / 2} \mathrm{Ba}_{1 / 2} \mathrm{MnO}_{3}$. Using the observed diffraction spots, we performed crystal-structure analyses. Comparisons between observed and calculated structure factors are shown in Fig. 1(a, b) Schematic views of the atomic displacements in the ferroelectric and multiferroic phases are shown in Fig. 1(c, d). To understand the effect of the magnetic order on the ferroelectricity in the multiferroic phase, we simulate the ferroelectric polarization in the ground-state G-type antiferromagnetic (G-AFM) structure. In the multiferroic phase, we consider two mechanisms to induce the ferroelectric polarization: hybridization between Mn $3 d$ and apical O2 $2 p$ states $\left(P_{\text {hyb }}\right)$ and in-plane Mn-O1-Mn magnetic exchange striction $\left(P_{\text {extr }}\right)$, as shown in Fig. 1(e). In G-AFM, the magnetic exchange striction prevents the atomic displacement of the side $\mathrm{O} 1$ ion, so that total ferroelectric polarization is reduced. We conclude that only positive $P_{\text {hyb }}$ contributes to the large ferroelectric polarization in the paramagnetic phase. In stark contrast, the magnetic exchange striction induces negative $P_{\text {extr }}$, causing the suppression of the ferroelectric polarization in the multiferroic phase.

[1] M. Dawber, et al., Rev. Mod. Phys. 77, 1083 (2005).

[2] R. E. Cohen, Nature (London) 358, 136 (1992).

[3] T. Kimura, et al., Nature (London) 426, 55 (2003).

[4] H. Sakai, et al., Phys. Rev. Lett. 107, 137601 (2011).

[5] D. Okuyama, et al., Phys. Rev. Research 2, 033038 (2020).

Keywords: Multiferroic, Crystal structure analysis, Manganite

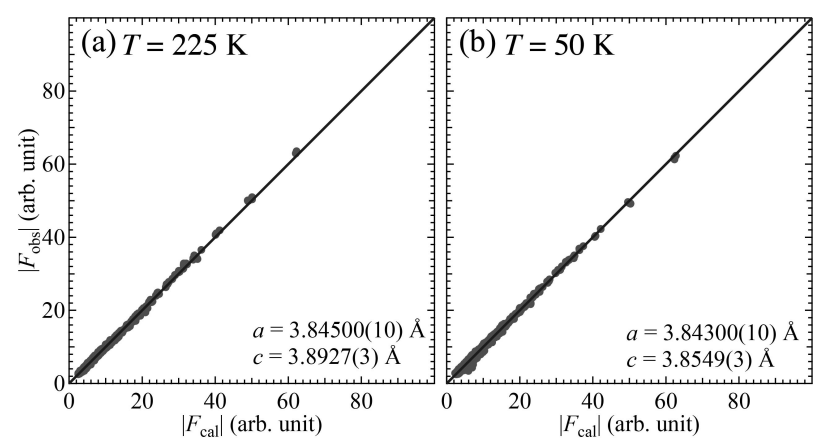

(c) $T=225 \mathrm{~K}$

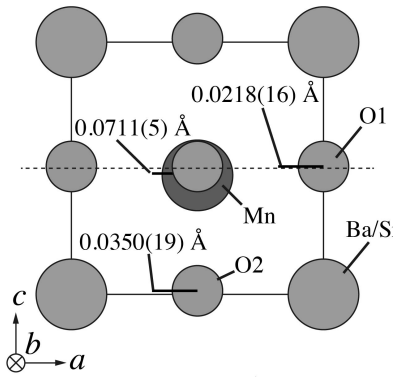

(d) $T=50 \mathrm{~K}$

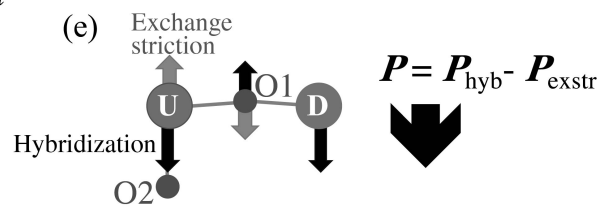

Figure 1. (a, b) Comparison between observed and calculated structure factors at $225 \mathrm{~K}$ in the ferroelectric phase and at $50 \mathrm{~K}$ in the multiferroic phase of tetragonal perovskite $\mathrm{Sr}_{1 / 2} \mathrm{Ba}_{1 / 2} \mathrm{MnO}_{3}$. (c, d) The ferroelectric atomic displacements at $225 \mathrm{~K}$ and $50 \mathrm{~K}$. (e) Schematic of ionic distortion and ferroelectric polarization for G-AFM. $P_{\text {hyb }}$ and $P_{\text {exstr }}$ stand for the ferroelectric polarization from the hybridization and the exchange striction, respectively. $U$ and $\mathrm{D}$ denote up- and down-spin Mn sites, respectively. 